\title{
A Qualitative Study on Barriers to Treatment and Control of Hypertension Among Patients at Dessie Referral Hospital, Northeast Ethiopia, Ethiopia: Healthcare Workers' Perspective
}

\author{
Yeshewas Abaynew (1D' \\ Mohammed Hussien $\mathbb{D}^{2}$ \\ 'School of Public Health, College of \\ Medicine and Health Sciences, Wollo \\ University, Dessie, Ethiopia; ${ }^{2}$ Department \\ of Health Systems Management and \\ Health Economics, School of Public \\ Health, College of Medicine and Health \\ Sciences, Bahir Dar University, Bahir Dar, \\ Ethiopia
}

Introduction: Hypertension is the leading cause of morbidity and mortality and accounts for $13 \%$ of all deaths and $7 \%$ of the disease burden in the world. Although the importance of controlling hypertension has been recognized for many years, the majority of patients with hypertension remain uncontrolled. Studies indicate a very low adherence to lifestyle modifications among patients in the study area. Hypertensive patients face set of problems associated with adherence to antihypertensive drugs and healthy lifestyle modifications.

Objective: The objective of the study was to explore barriers to hypertension treatment and control from healthcare workers' perspective and experiences at Dessie Referral Hospital, Ethiopia. Methods: Seven healthcare workers actively involved in managing hypertensive individuals at Dessie Referral Hospital were recruited for the study. Participants were purposively selected from outpatient, inpatient, and pharmacy departments. The required data were collected by the principal investigator on exit interviews. An interview guide was developed by reviewing previous literature. Interviews were semi-structured, 8-20 minutes in duration, and designed to elicit healthcare workers'experiences and perceptions and conducted until data saturation was reached. All interviews were recorded, transcribed verbatim, and thematically analyzed. Each theme was supported by using the participants' quotes.

Results: Three themes emerged. The major barriers impacted patients' medication adherence and healthy lifestyle modifications were common use of diets during social settings, low level of awareness, lack of resources, misconceptions about hypertension, use of traditional medicines, inadequate physical activities, and high cost of drugs.

Conclusion: Patients' misconceptions about hypertension, common use of diets during festivals, and inadequate physical activities were factors associated with inadequate blood pressure control. Healthcare workers should better understand the problems that hypertensive patients' face, thus achieving better control.

Keywords: hypertension, adherence, healthcare workers, barriers, control, perspective

\section{Introduction}

Hypertension is the leading cause of morbidity and mortality, and accounts for $13 \%$ of all deaths and $7 \%$ of the disease burden in the world. ${ }^{1,2}$ Studies have shown that interventions for the treatment and control of hypertension were poorly implemented in hypertensive patients ${ }^{3-5}$ and the prevalence of uncontrolled hypertension remains high. ${ }^{6-8}$ A prevalence of $43.5 \%$ non-adherence to antihypertensive medications was reported. ${ }^{4}$ Moreover, inadequate treatment and poor adherence lead to
Correspondence: Yeshewas Abaynew School of Public Health, College of Medicine and Health Sciences, Wollo University, Dessie, Ethiopia Email yag2005@yahoo.com 
long-term complications such as stroke, health failure, and chronic kidney disease, ${ }^{9,10}$ and hospitalization. ${ }^{11}$

Despite the significant advances in pharmacological approaches to treat hypertension, the rates of hypertension treatment and control continue to be low in the world, particularly in low-to-middle-income countries. ${ }^{12,13}$ Evidences showed that there are patients who do not follow the medical or lifestyle recommendations. ${ }^{14,15}$ In developing countries, $66-70 \%$ of the patients did not appropriately control their blood pressure. ${ }^{16}$

In a study conducted among hypertensive patients in Ghana and Nigeria, poor blood pressure control was observed in $69.7 \%$ of the study participants. ${ }^{17}$ A study conducted in Kilimanjaro revealed that only $10 \%$ of the hypertensive patients are on medication and less than $1 \%$ had optimal controlled blood pressure. ${ }^{18}$ Studies conducted in Ethiopia reported variable magnitude.

Adherence to medication and lifestyle changes is a problem in chronic diseases management and failure to adhere is associated with reduced patient's quality of life, increased expense to the healthcare system, ${ }^{10}$ the occurrence of medical and psychological complications of the disease, ${ }^{19}$ and poses a significant burden on healthcare utilization. ${ }^{20}$ A study done in Northern Sri Lanka indicated that most patients $(84.5 \%)$ had poor drug compliance. ${ }^{21}$ A cross-sectional study among Palestinian hypertensive patients reported that more than half of patients (54.2\%) did not take their antihypertensive medications. ${ }^{10} \mathrm{~A}$ crosssectional study done at University of Gondar Hospital, Northwest Ethiopia revealed that $34.5 \%$ of the study participants were found to be non-adherent to their treatment. ${ }^{19}$ A study done in Northern Ethiopia indicated that around one-fourth (26.4\%) of the study participants were found to be non-adherent to their treatment. ${ }^{22}$ Additionally, studies done in Ethiopia found an overall prevalence of anti-hypertensive medication non adherence to be $26 \%,{ }^{23} 16.5 \%,{ }^{24}$ and $48.1 \% .{ }^{25}$ Another study conducted in Dessie Referral Hospital, Ethiopia, ${ }^{26}$ reported a very low adherence $(23.6 \%)$ to lifestyle modifications among hypertensive patients.

Barriers to inadequate practices of the recommendations of hypertension control might be related to the patients, healthcare workers, and healthcare system ${ }^{27}$ including lack of awareness of the condition, patient's belief that hypertension is treatable and the medicine should be taken for lifelong, poor adherence to medication, low motivation for treatment and lifestyle changes, peer pressure for smoking and drinking, food culture, and lack of social support. ${ }^{2}$ In addition, a shortage of equipment, staff, space, providers' lack of time for consultation, communication skills, economic barriers, stress, the use of traditional remedies, misconceptions, low community awareness, lack of patient knowledge, cultural beliefs, psychosocial factors, high medication costs, and drug side effects were commonly reported barrier by providers. ${ }^{2,14,28}$

Studies that have qualitatively assessed reasons for low hypertension treatment, and control are minimal in lowand lower-middle-income countries. ${ }^{29}$ This study used a qualitative method to explore the barriers of treatment and control of hypertension in Ethiopia.

Hence, this study will provide a better understanding of the reasons why hypertensive patients do not follow treatment and healthy lifestyle recommendations.

\section{Methods}

A qualitative approach was used to explore healthcare workers' perspectives and experience regarding managing hypertensive patients in order to identify the main reasons patients faced to adhere to the recommendations to control blood pressure.

The study was conducted at Dessie Referral Hospital, which is located in Dessie, Amhara Regional state, which is located at 401 kilometers from the capital of Ethiopia, Addis Ababa, and 480 kilometers away from the regional city, Bahir Dar. There are two public hospitals, and three private hospitals.

Purposive sampling was used to select the healthcare workers from outpatient, inpatient, and pharmacy departments where hypertensive patients have had contact during receiving antihypertensive management.

The interview guide (Supplementary material) was piloted in BoruMeda Hospital and necessary amendment was made based on the results like rearranging the ordering of the questions. Data were collected from seven healthcare workers who were actively involved in managing hypertensive patients in the hospital. Two medical doctors, three nursing professionals, and two pharmacy professionals were included in the study. All of the study participants were male. The study participants have $2-10$ years of clinical experiences. Two female participants who are working in the inpatient department refused to participate in the study due to lack of time for the interview. But male healthcare workers working in the inpatient department were invited to take part in the study 
and they were agreed to be interviewed in place of females who refused to participate.

Face-to-face semi-structured interviews were conducted for data collection. The qualitative interview guide was developed after an extensive literature review. During the interview, healthcare workers were asked probing questions to provide further details on their responses. The data were gathered from the study participants until data saturation was reached and no new further findings were drawn from the interview. The data were collected from December 4 to 8, 2017.

The interviews were conducted by the principal investigator. The discussions were audio recorded and field notes were taken during an interview. The interview lasted approximately from 8 to 20 minutes. The interview was conducted at a private room. Interviews were conducted until saturation was reached.

The audio-recorded data from interviews of healthcare workers were transcribed verbatim. The transcripts of the study were returned to study participants for comment and correction. After review, participants provided their feedback on the findings to enrich the transcripts. Data analysis was done iteratively with the adjustment of the content of the questions. The final transcripts were thematically analyzed for their contents. The themes were derived from the data during analysis. Direct quotes of participants that illustrated important themes were extracted and included in the result. Software was not used for data analysis.

Ethical approval was granted from the Research Ethics Committee of College of Medicine and Health Sciences of Wollo University. Permission was obtained from the authority of the Hospital to conduct the study. Prior to the commencement of the interview, the study was briefed to the participants and verbal informed consent was obtained. An informed consent including publication of anonymized responses was secured from study participants.

After receiving verbal informed consent, the study participants were interviewed in a private room. By assuming the issue under study was not much sensitive, written informed consent was not requested from study participants. With participants' permission, the interview was audio-taped. Furthermore, transcriptions were stored and managed privately to ensure confidentiality.

\section{Results}

Healthcare workers who are currently managing hypertensive patients were interviewed for the study. Data saturation was attained after interviewing the seventh healthcare workers. Healthcare professional reported their patients' challenges and difficulties in the management of hypertension and additionally they also reported their own view on the challenges patients faced in hypertension prevention and control. This study indicated key themes that can be used in changing the beliefs and experiences of hypertensive patients. During the analysis, three main themes were identified. Themes and selected quotations were organized as follows.

\section{Theme I: Barriers to Antihypertensive Medication Adherence}

Healthcare workers reported that some patients did not take antihypertensive medication as prescribed. There were reasons mentioned by the healthcare workers for non-adherence of patients to their drugs: Some patients felt that medication for hypertension is unnecessary because they believed that blood pressure should be better controlled by lifestyle changes such as dietary change. The other reasons stated were faith in traditional medicine, stress, forgetfulness, afraid of drugs side effects, afraid of becoming dependent on medication, discontinuing taking drugs when achieving normal blood pressure, lack of support from family and community, cost of drugs, and shortages of resources.

Patients didn't take their prescribed drugs appropriately because they prefer traditional medicine for controlling their blood pressure. (Medical doctor, 3 years of experience)

Patients reported that they stopped taking drugs to control their blood pressure when they realized that their blood pressure was lowered to the expected level because they were thought that they were cured from hypertension. (Nursing professional, 3 years of experience)

Sometimes antihypertensive drugs are not available in the hospital. When there was a shortage of drugs, patients were forced to buy drugs with high cost from private pharmacies. It was difficult for some patients to afford it. (Medical doctor, 3 years of experience)

There were shortages of resources such as rooms to manage hypertensive cases in the hospital. It was difficult to counsel patients with the existing small number of rooms. We could not provide adequate information about hypertension and its management because of such shortages (Nursing professional, 3 years of experience) 


\section{Theme 2: Barriers to Adherence to Lifestyle Modifications (Physical Activity + Diet Changes)}

Healthcare workers stated that there were barriers patients faced to follow the recommendations for managing their blood pressure. The barriers related to the non-adherence of patients to the recommendation of the needs of physical activity and diet change to control their blood pressure were lack of self-motivation to exercise, low level of awareness of the community about the disease, the type of food and beverages available at social events, less common practice of physical activity in the community, shortage of time, and social influences.

A medical doctor with 2 years of experience stated,

Patients reported that they could not find their preferred healthy food and drinking to eat and drink during attending social events. Because for festivals salty and oily foods were prepared for all peoples invited to celebrate the festivals. Food preparations for festivals did not consider hypertensive patients' food chose. If even patients were aware of the unhealthy food preparations, they were forced to consume the food prepared for the whole people attended the festivals. Because they said that they feared social isolation from the community if they did not decide to eat like the rest of the people.

A reason reported by patients for not doing regular physical activity for controlling their blood pressure was that it was not a common practice to do physical activity in the community. It was not a habitual behavior in the community so that it was impossible to do physical activity in such a community. (Nursing professional, 3 years of experience)

Healthcare workers usually said few words to patients and did not give full information about the disease and its management like what would happen in the future if they didn't take their drugs and the need to strictly follow the medical recommendations because healthcare workers did not have much time to provide the full information in need. (Pharmacy professional, 9 years of experience)

\section{Theme 3: Suggestions to Improve Hypertension Control}

Healthcare workers stated that adequate support of families/community, increasing awareness about hypertension; adequate consultation session and availability of adequate healthcare resources were needed to improve patients' medication adherence and behavioral changes to lifestyle modifications to control hypertension.

Providing adequate counseling session to hypertensive patients is encouraging to the patients in order to take drugs appropriately, follow lifestyles modifications. Therefore, I recommended that healthcare workers should lengthen the counseling session for hypertensive patients to provide accurate and full information that would facilitate the medication adherence and behavioral modification. (Nursing professional, 10 years of experience)

\section{Discussion}

This study found that low level of awareness, fear of drug dependency, reluctance to take drugs, use of traditional medicines, fear of drug side effects, shortage of resources such as lack of drugs, misconceptions, common use of diets during social settings, lack of doing physical exercise, stress, and cost of drugs were the major barriers patients faced during treatment and control of hypertension. In addition, the study highlighted areas that need improvement related to medication adherence and lifestyle changes including the need to provide adequate support and counselling.

The aforementioned findings are supported by previous study, ${ }^{28}$ which stated that economic constraints, stress, and use of traditional remedies such as herbal medicine, medication side effects, and low community awareness as barriers to hypertension control. Similarly, a study done in Uzbekistan showed that switching to traditional therapy, being afraid of addiction, costs, availability of drugs and duration of therapy were strong reasons for nonadherence. ${ }^{16}$ Moreover, in a study done in Sri Lanka barriers such as cultural practices, adverse drug reactions, drug out of supply, and expenses were reported as reasons for non-adherence of drugs. ${ }^{21}$ Similarly, another study findings showed that the most common predictors of poor drug adherence were poor knowledge, negative perception about medication, side effects, feared dependence, lack of availability and high medication cost. ${ }^{16} \mathrm{~A}$ prior qualitative study also showed that reluctance or hesitancy to make a commitment to lifelong medication and financial barriers (cost) were associated with blood pressure medication non-adherence. ${ }^{30}$ Additionally, a study from India ${ }^{29}$ indicates that the cost of medicines as a barrier for low adherence to antihypertensive medication in women. Other researchers also reported cost as a major barrier for some patients. ${ }^{31,32}$ 
The present study reveals weak patient-healthcare provider interactions and fear of drug side effects as major barrier to hypertension control. Similarly, a study done in Ethiopia $^{23}$ reported healthcare provider poor interaction with patient, frustration and unwanted effect of the medication in the long run as major causes of non-compliant to medication among hypertensive patients. Additionally, lack of physical exercise is reported a major problem among hypertensive patients. This finding is supported by a study done in Ethiopia, ${ }^{26}$ which reported that more than half of the patients did not perform physical exercise, $60.1 \%$ of non-adherent to exercise. Regarding adherence to diet recommendation, a study done in Ethiopia ${ }^{26}$ also support the finding of this study, which shows a $23.6 \%$ non-adherence to diet-related recommendations.

This study applied a qualitative design to explore the insights of healthcare workers selected from different departments in order to accommodate the various levels of healthcare workers' experiences in managing hypertensive patients, which is the strength of the current study. Moreover, patients, families and community perspectives were not obtained directly addressed in the study that might be the limitation of the study.

\section{Conclusions}

Barriers to hypertension treatment and control were multifactorial. Misconceptions about hypertension, common use of diets during festivals, and inadequate physical activities were factors associated with inadequate blood pressure control. In order to improve hypertensive patients' adherence to treatment and control strategies, healthcare workers should provide adequate information with structured counseling sessions to increase patients' awareness about hypertension. Conducting health education campaigns in the community to disseminate appropriate health information to increase public knowledge and awareness about hypertension is also recommended. There is a need for healthcare workers to be aware of patients' misbeliefs that decreased medication adherence and healthy lifestyle changes, and further study is also advised to be done from patients and families perspective.

\section{Data Sharing Statement}

All data generated or analyzed in the study are included in this manuscript.

\section{Acknowledgments}

The authors acknowledged study participants and hospital management staff for their willingness and cooperation to conduct the study.

\section{Author Contributions}

Both authors contributed to data analysis, drafting or revising the article, have agreed on the journal to which the article was submitted, gave final approval of the version to be published, and agree to be accountable for all aspects of the work.

\section{Funding}

No funding was received for this study.

\section{Disclosure}

The authors report no conflicts of interest for this work.

\section{References}

1. World Health Organization. Global Health Risks; Mortality and Burden of Disease Attributable to Selected Major Risks. Geneva: World Health Organization; 2009.

2. Shrestha S, Shrestha A, Koju RP, et al. Barrier and facilitators to treatment among newly diagnosed hypertensive patients in Nepal: a Qualitative Study. Heart Asia. 2018;7(34):1-11.

3. Okwuonu CG, Emmanuel CI, Ojimadu NE. Perception and practice of lifestyle modification in the management of hypertension among hypertensives in south-east Nigeria. Int J Med Biomed Res. 2014;3 (2):121-131.

4. Bhandari B, Bhattarai M, Ghimire A, Pokharel P. Adherence to antihypertensive medications: population based follow up in adherence to antihypertensive medications: population based follow up in Eastern Nepal. J Nepal Health Res Counc. 2015;13(29):38-42.

5. Okai DE, Manu A, Amoah EM, Laar A, Akamah J, Torpey K. Patient-level factors influencing hypertension control in adults in Accra, Ghana. BMC Cardiovasc Disord. 2020;20(1):123. doi:10.1186/s12872-020-01370-y

6. Kearney PM, Whelton M, Reynolds K, Muntner P, Whelton PK, He J. Global burden of hypertension: analysis of worldwide data. Lancet. 2005;365(9455):217-222. doi:10.1016/S0140-6736(05) 17741-1

7. Chow CK, Teo KK, Rangarajan S, et al. Prevalence, awareness, treatment, and control of hypertension in rural and urban communities in high-, middle-, and low-income countries. JAMA. 2013;310 (9):959-968. doi:10.1001/jama.2013.184182

8. Saju MD, Allagh KP, Scaria L, Joseph S, Thiyagarajan JA. Prevalence, awareness, treatment, and control of hypertension and its associated risk factors: results from baseline survey of SWADES Family Cohort Study. Int J Hypertens. 2020;2020:1-7. doi:10.1155/ 2020/4964835

9. Vrijens B, Kristanto P, Urquhart J, Burnier M. Adherence to prescribed antihypertensive drug treatments: longitudinal study of electronically compiled dosing histories. BMJ. 2008;1-6. doi:10.1136/ bmj.39416.559942.BE

10. Al-Ramahi R. Adherence to medications and associated factors: a cross-sectional study among Palestinian hypertensive patients. $J$ Epidemiol Glob Health. 2014;5(2):125. doi:10.1016/j. jegh.2014.05.005 
11. Tsadik DG, Berhane Y, Worku A. Adherence to antihypertensive treatment and associated factors in central Ethiopia. Int J Hypertens. 2020;2020:10-13.

12. Vedanthan R, Tuikong N, Kofler C, et al. Barriers and facilitators to nurse management of hypertension: a qualitative analysis from Western Kenya. Ethn Dis. 2016;26(3):315-322. doi:10.18865/ ed.26.3.315

13. Park JE, Park J, Chang SJ, Lee JH, Kim SY. The determinants of and barriers to awareness and treatment of hypertension in the Korean Population. Asia Pac J Public Health. 2019;31(2):121-135.

14. Jolles EP, Padwal RS, Clark AM, Braam B. A qualitative study of patient perspectives about hypertension. Hypertension. 2013;2013. doi:10.5402/2013/671691

15. Shima R, Farizah MH, Majid HA. A qualitative study on hypertensive care behavior in primary health care settings in Malaysia. Patient Prefer Adherence. 2014;8:1597-1609. doi:10.2147/PPA.S69680

16. Malik A, Yoshida Y, Erkin T, Salim D, Hamajima N. Hypertensionrelated knowledge, practice and drug adherence among inpatients of a hospital in Samarkand, Uzbekistan. J Med Sci. 2014;76:255-263.

17. Boima V, Ademola AD, Odusola AO, et al. Factors associated with medication nonadherence among hypertensives in Ghana and Nigeria. Int J Hypertens. 2015;2015:1-8. doi:10.1155/2015/205716

18. Muhamedhussein MS, Nagri ZI, Manji KP. Prevalence, risk factors, awareness, and treatment and control of hypertension in Mafia Island, Tanzania. Int $J$ Hypertens. 2016;2016:1-5. doi:10.1155/2016/ 1281384

19. Ambaw AD, Alemie GA, Yohannes SMW, Mengesha ZB. Adherence to antihypertensive treatment and associated factors among patients on follow up at University of Gondar Hospital, Northwest Ethiopia. BMC Public Health. 2012;12(1):1. doi:10.1186/1471-2458-12-282

20. Saleem F, Ma H, Aa S, Atif M. Drug attitude and adherence: a qualitative insight of patients with hypertension. J Young Pharm. 2012;4(2):101-107. doi:10.4103/0975-1483.96624

21. Pirasath S, Kumanan T, Guruparan M. A study on knowledge, awareness, and medication adherence in patients with hypertension from a tertiary care centre from northern Sri Lanka. Int J Hypertens. 2017;2017:1-6. doi:10.1155/2017/9656450

22. Ali MA, Bekele ML, Teklay G. Antihypertensive medication non-adherence and its determinants among patients on follow up in public hospitals in Northern Ethiopia. Int J Clin Trials. 2014;1 (3):95-104. doi:10.5455/2349-3259.ijct20141103
23. Chelkeba L, Dessie S. Antihypertension medication adherence and associated factors at Dessie. Int J Res Med Sci. 2013;1(3):125-127. doi:10.5455/2320-6012.ijrms20130802

24. Wake AD, Tuji TS, Sime AT, Mekonnin MT, Taji TM, Hussein AA. Medications, and associated factors among hypertensive patients in a follow-up clinic at asella referral and teaching hospital, Ethiopia: a Cross-Sectional Study. Int $J$ Hypertens. 2021;2021:1-10. doi:10.1155/2021/7359318

25. Andualem A, Liknaw T, Edmealem A, Gedefaw M. Adherence to antihypertensive medications among adult hypertensive patients attending chronic follow-up units of Dessie referral hospital, Northeastern Ethiopia. Medicine. 2021;100(31):e26818. doi:10.1097/MD.0000000000026818

26. Andualem A, Gelaye H, Damtie Y. Adherence to lifestyle modifications and associated factors among adult hypertensive patients attending chronic follow-up units of Dessie referral hospital, north east Ethiopia. Integr Blood Press Control. 2020;2015:145-156. doi:10.2147/IBPC.S275575

27. Ogedegbe G. Barriers to optimal hypertension control. J Clin Hypertens. 2008;10(8):1-5. doi:10.1111/j.1751-7176.2008.08329.x

28. Gebrezgi MT, Trepka MJ, Kidane EA, Gebrezgi MT, Trepka MJ, Kidane EA. Barriers to and facilitators of hypertension management in Asmara, Eritrea: patients' perspectives. J Health Popul Nutr. 2017;36(1):11. doi:10.1186/s41043-017-0090-4

29. Gupta S, Dhamija JP, Mohan I, Gupta R. Qualitative study of barriers to adherence to antihypertensive medication among rural women in India. Int J Hypertens. 2019;2019:1-7. doi:10.1155/2019/5749648

30. Johnson HM, Warner RC, Bartels CM, Lamantia JN. "They' re younger ... it' s harder." Primary providers' perspectives on hypertension management in young adults: a multicenter qualitative study. BMC Res Notes. 2017;1-9. doi:10.1186/s13104-016-2345-3

31. Howes F, Hansen E, Williams D, Nelson M. Barriers to diagnosing and managing hypertension A qualitative study in Australian general practice. Aust Fam Physician. 2010;39(7):511-516.

32. Hong SH, Wang J, Tang J. Dynamic view on affordability of fixed-dose combination antihypertensive drug therapy. $\mathrm{Am}$ J Hypertens. 2013;26(7):879-887. doi:10.1093/ajh/hpt035
Integrated Blood Pressure Control

\section{Publish your work in this journal}

Integrated Blood Pressure Control is an international, peer-reviewed open-access journal focusing on the integrated approach to managing hypertension and risk reduction. Treating the patient and comorbidities together with diet and lifestyle modification and optimizing healthcare resources through a multidisciplinary team approach constitute key features of the journal. This journal is indexed on

\section{Dovepress}

American Chemical Society's Chemical Abstracts Service (CAS). The manuscript management system is completely online and includes a very quick and fair peer-review system, which is all easy to use. Visit http://www.dovepress.com/testimonials.php to read real quotes from published authors. 